\title{
$k$-SPACES AND PRODUCTS OF CLOSED IMAGES OF METRIC SPACES
}

\author{
GARY GRUENHAGE
}

\begin{abstract}
We show that a recent theorem of Y. Tanaka giving necessary and sufficient conditions for the product of two closed images of metric spaces to be a $k$-space is independent of the usual axioms of set theory.
\end{abstract}

1. Introduction. According to $Y$. Tanaka $\left[T_{1}\right]$, a space $X$ is said to belong to class $\mathfrak{I}^{\prime}$ if it is the union of countably many closed and locally compact subsets $X_{n}$ such that $A \subset X$ is closed whenever $A \cap X_{n}$ is closed in $X_{n}$ for all $n=1,2, \ldots$ In a later paper $\left[\mathrm{T}_{3}\right]$, Tanaka, assuming the continuum hypothesis $(\mathrm{CH})$, gives necessary and sufficient conditions for the product of two closed images of metric spaces to be a $k$-space:

TheOREM (CH). Let $X$ and $Y$ be closed images of metric spaces. Then $X \times Y$ is a $k$-space if and only if one of the following holds:

(1) $X$ and $Y$ are metric spaces;

(2) $X$ or $Y$ is a locally compact metric space;

(3) $X$ and $Y$ are in the class $\mathfrak{I}^{\prime}$.

Of course, it is natural to ask if the assumption of $\mathrm{CH}$ is necessary. In this paper, we shall show that this theorem is in fact equivalent to a certain set-theoretic axiom weaker than $\mathrm{CH}$. At first glance, this may seem a bit odd. But it turns out that the truth of the theorem depends on its truth for a very special class of spaces. If $\sigma$ is a cardinal number, let $S_{\sigma}$ be the space obtained from the disjoint union of $\sigma$ convergent sequences by identifying all the limit points to a single point. We will show that the truth of the theorem depends on whether or not $S_{\omega} \times S_{\omega_{1}}$ is a $k$-space. It is not so surprising that this depends on your set theory.

2. Main results. We use the following conventions. If $A$ and $B$ are sets, then ${ }^{A} B$ is the set of all functions from $A$ into $B$. Cardinals are initial ordinals, and an ordinal is the set of its predecessors.

For two functions $f$ and $g$ from $\omega$ to $\omega$, we define $f \leqslant g$ if and only if the set $\{n \in \omega: f(n)>g(n)\}$ is finite. If $\kappa$ is a cardinal number, then $\operatorname{BF}(\kappa)$ is the following assertion.

$\mathrm{BF}(\kappa)$ : If $F \subset^{\omega} \omega$ has cardinality less than $\kappa$, then there exists $g \in{ }^{\omega} \omega$ such that $f<g$ for all $f \in F$.

Received by the editors September 26, 1979; presented to the Society, January 25, 1980. 1980 Mathematics Subject Classification. Primary 54D50; Secondary 54B10, 54C10.

Key words and phrases. $k$-space, $\mathrm{BF}(\boldsymbol{\kappa})$. 
It is known that Martin's Axiom implies that $\mathrm{BF}(\kappa)$ holds for all $\kappa$ less than or equal to the continuum. It is easy to observe that $\mathrm{CH}$ implies that $\mathrm{BF}\left(\omega_{2}\right)$ is false. Applying $\mathrm{BF}(\kappa)$ to topological problems is not new-see [vDW], for example. The following lemma demonstrates why $\mathrm{BF}(\kappa)$ is of interest to us.

LEMMA 1. Let $\kappa$ be a cardinal number, and let $\kappa^{+}$denote the least cardinal greater than $\kappa$. Then $S_{\omega} \times S_{\kappa}$ is a $k$-space (or a sequential space) if and only if $\mathrm{BF}\left(\kappa^{+}\right)$.

Proof. Suppose $\mathrm{BF}\left(\kappa^{+}\right)$is false. Then there exists a collection $\left\{f_{\alpha} \in{ }^{\omega} \omega: \alpha<\kappa\right\}$ such that if $f \in{ }^{\omega} \omega$, then there exists $\alpha<\kappa$ with $f_{\alpha}(n)>f(n)$ for infinitely many $n \in \omega$.

For each $\alpha<\kappa$, let $H_{\alpha}=\left\{\left(m_{n}, n_{\alpha}\right) \in S_{\omega} \times S_{\kappa}: m<f_{\alpha}(n)\right\}$, where $m_{n}$ and $n_{\alpha}$ denote the $m$ th term of the $n$th sequence in $S_{\omega}$ and the $n$th term of the $\alpha$ th sequence in $S_{\kappa}$, respectively. Let $H=\cup_{\alpha<\kappa} H_{\alpha}$.

We will prove that $H$ is $k$-closed, but not closed. If $K$ is a compact set in $S_{\omega} \times S_{\kappa}$, then $K$ meets only finitely many sequences in each factor. Thus $K$ meets only finitely many $H_{\alpha}$ 's. Since each $H_{\alpha}$ is closed, $K \cap H$ is closed. Thus $H$ is $k$-closed. Now let $\infty$ denote the nonisolated point in $S_{\omega}$ and $S_{\kappa}$. Suppose $U$ is any open set in $S_{\omega} \times S_{\kappa}$ containing $(\infty, \infty)$. Let $f \in{ }^{\omega} \omega$ and $g \in{ }^{\kappa} \omega$ be such that $\left(m_{k}, n_{\alpha}\right) \in U$ whenever $f(k) \leqslant m$ and $g(\alpha)<n$. There exists $\alpha<\kappa$ such that $f_{\alpha}(n)>f(n)$ for infinitely many $n \in \omega$. Thus there exists $n^{\prime} \in \omega$ such that $g(\alpha)<n^{\prime}$ and $f_{\alpha}\left(n^{\prime}\right)>f\left(n^{\prime}\right)$. This implies that $\left(f_{\alpha}\left(n^{\prime}\right)_{n^{\prime}}, n_{\alpha}^{\prime}\right) \in H_{\alpha} \cap U$. So we have shown that $(\infty, \infty) \in \bar{H} \backslash H$, and yet $H$ is $k$-closed. Thus $S_{\omega} \times S_{\kappa}$ is not a $k$-space. This proves the "only if" part of Lemma 1.

Now assume $\mathrm{BF}\left(\kappa^{+}\right)$holds. Suppose $H$ is a subset of $S_{\omega} \times S_{\kappa}$ which is sequentially closed, but not closed. Then $\left(\left\{n_{m}\right\} \times S_{\kappa}\right) \cap H$ and $\left(S_{\omega} \times\left\{n_{\alpha}\right\}\right) \cap H$ are closed for each $n_{m} \in S_{\omega}$ and $n_{\alpha} \in S_{\kappa}$, so $\bar{H}=H \cup\{(\infty, \infty)\}$. Also, $H \cap[(\{\infty\} \times$ $\left.\left.S_{\kappa}\right) \cup\left(S_{\omega} \times\{\infty\}\right)\right]$ is closed (if not, $(\infty, \infty)$ would be a sequential limit point of $H$ ), so we may assume witout loss of generality that $H$ contains only isolated points.

If $f \in{ }^{\omega} \omega$, let $U_{f}$ be the open set in $S_{\omega}$ defined by $U_{f}=\{\infty\} \cup\left\{m_{n}: m \geqslant f(n)\right\}$. For $g \in{ }^{\kappa} \omega$, define analogously an open set $V_{g}$ in $S_{\kappa}$.

Since $S_{\omega} \times\left(\left\{n_{\alpha}: n \in \omega\right\} \cup\{\infty\}\right)$ is sequential for fixed $\alpha$, there exists $f_{\alpha} \in{ }^{\omega} \omega$ and $n(\alpha) \in \omega$ such that $\left(U_{f_{\alpha}} \times\left\{m_{\alpha}: m>n^{\prime}(\alpha)\right\}\right) \cap H=\varnothing$. Applying $\operatorname{BF}\left(\kappa^{+}\right)$, there exists a function $f$ which bounds all the $f_{\alpha}$ 's, $\alpha<\kappa$. For each $\alpha$, let $k(\alpha) \in \omega$ be such that $f_{\alpha}(k)<f(k)$ whenever $k \geqslant k(\alpha)$.

For each $\alpha<\kappa$, there exists $n^{\prime}(\alpha) \in \omega$ with $n^{\prime}(\alpha)>n(\alpha)$ such that $\left(\left(\cup_{j<k(\alpha)}\left\{m_{j}\right.\right.\right.$ : $\left.m \in \omega\}) \times\left\{m_{\alpha}^{\prime}: m^{\prime} \geqslant n(\alpha)\right\}\right) \cap H=\varnothing$, for otherwise $(x, \infty)$ would be a sequential limit point of $H$ for some $x \in S_{\omega}$. Now define $g \in{ }^{\kappa} \omega$ by $g(\alpha)=n^{\prime}(\alpha)$. Suppose $\left(m_{n}, m_{\alpha}^{\prime}\right) \in U_{f} \times V_{g}$. If $n<k(\alpha)$, then since $m^{\prime}>n^{\prime}(\alpha)$, it is true that $\left(m_{n}, m_{\alpha}^{\prime}\right) \notin$ $H$. If $n>k(\alpha)$, then $f_{\alpha}(n) \leqslant f(n)<m$, and so $\left(m_{n}, m_{\alpha}^{\prime}\right) \in U_{f_{\alpha}} \times\left\{p_{\alpha}: p>n(\alpha)\right\}$, which implies $\left(m_{n}, m_{\alpha}^{\prime}\right) \notin H$. Thus $\left(U_{f} \times V_{g}\right) \cap H=\varnothing$, contradicting the assumption that $H$ was not closed. It follows that $S_{\omega} \times S_{\kappa}$ is sequential (and thus a $k$-space). 
A space $X$ is a Fréchet space if whenever $A \subset X$ and $x \in \bar{A}$, then there exists a sequence $x_{1}, x_{2}, \ldots$ contained in $A$ which converges to $x$.

LEMMA 2. Let $f: X \rightarrow Y$ be a closed map with $X$ a collectionwise-normal Fréchet space. Let $y \in Y$. If $\partial f^{-1}(y)$ contains a closed discrete subset of cardinality $\sigma$, then $Y$ contains a closed subset homeomorphic to $S_{\sigma}$.

Proof. Suppose $D$ is a closed discrete subset of $f^{-1}(y)$ of cardinality $\sigma$. Let $\left\{U_{d}\right.$ : $d \in D\}$ be a discrete collection of open sets with $d \in U_{d}$. For each $d \in D$, let $d_{0}$, $d_{1}, d_{2}, \ldots$ be a sequence in $U_{d} \backslash f^{-1}(y)$ converging to $d$, such that $f\left(d_{n}\right) \neq f\left(d_{n^{\prime}}\right)$ whenever $n, n^{\prime} \in \omega, n \neq n^{\prime}$. For each $d \in D$ and $n \in \omega$, let $E\left(d_{n}\right)=\left\{d^{\prime} \in D\right.$ : $f\left(d_{m}^{\prime}\right)=f\left(d_{n}\right)$ for some $\left.m \in \omega\right\}$. Since $f$ is closed, it is easy to see that for each $d \in D$, there exists $n(d)$ such that $\cup_{n=n(d)}^{\infty} E\left(d_{n}\right)$ is finite for every $d \in D$.

Now pick $d(0) \in D$. If $d(\alpha)$ has been defined for all $\alpha<\beta$, where $\beta<\sigma$, pick $d(\beta) \in D \backslash \cup_{\alpha<\beta} E(d(\alpha))$. Let $D^{*}=\{d(\alpha): \alpha<\sigma\} \cup\left\{d(\alpha)_{n}: \alpha<\sigma\right.$ and $n \in$ $\omega$ ). Then $D^{*}$ is a closed subset of $X$, and $f$ is one-to-one on $D^{*} \backslash f^{-1}(y)$. Thus $f\left(D^{*}\right)$ is homeomorphic to $S_{\sigma}$ and is closed in $Y$.

Now we describe another simple space which will have a role to play later on. For each $n \in \omega$, let $T_{n}$ be a countably infinite set. Let $T=\left(\cup_{n \in \omega} T_{n}\right) \cup\{\infty\}$, where the points of $T_{n}$ are isolated for each $n \in \omega$, and a basic open set containing $\infty$ has the form $\{\infty\} \cup\left(\cup_{n>k} T_{n}\right)$, where $k \in \omega$.

LEMMA 3. If $X$ is a regular first countable space and $X$ is not locally countably compact, then $X$ contains a closed subset homeomorphic to $T$.

Proof. We omit the straightforward proof of this lemma.

LEMMA 4. $S_{\omega} \times T$ is not a $k$-space.

PRoof. Let $t_{n, m}$ denote the $m$ th term in some enumeration of $T_{n}$. As before, let $m_{n}$ denote the $m$ th term of the $n$th sequence in $S_{\omega}$. Let $H=\left\{\left(m_{n}, t_{n, m}\right) \in S \times T\right.$ : $n, m \in \omega\}$. It is easy to check that $H$ is $k$-closed but not closed, and so $S \times T$ is not a $k$-space.

Now we are ready for the main result.

THEOREM 1. The following are equivalent:

(a) $S_{\omega} \times S_{\omega_{1}}$ is not a $k$-space;

(b) $\mathrm{BF}\left(\omega_{2}\right)$ is false;

(c) if $X$ and $Y$ are closed images of metric spaces, then $X \times Y$ is a $k$-space if and only if one of the following holds:

(i) $X$ and $Y$ are metrizable;

(ii) $X$ or $Y$ is locally compact and metrizable;

(iii) $X$ and $Y$ are in the class $\mathfrak{I}^{\prime}$.

Proof. We have (a) $\leftrightarrow$ (b) from Lemma 1 . It is easy to check that $S_{\omega_{1}}$ is not in class $\mathfrak{I}^{\prime}$. So we also have (c) $\rightarrow$ (a). Assume (a) holds. Tanaka's proof of the "if" part of (c) does not use any axioms of set theory beyond ZFC, so we just need to prove the "only if" part. To this end, assume $X$ and $Y$ are closed images of metric 
spaces, and that $X \times Y$ is a $k$-space. If $X$ and $Y$ are closed $s$-images (i.e., point-inverses are separable) of metric spaces, then Tanaka [ $\left.\mathbf{T}_{1}\right]$ has shown that (i), (ii), or (iii) holds. So assume $X$ is not a closed $s$-image of a metric space. Then by Lemma 2, $X$ contains a closed subset homeomorphic to $S_{\omega_{1}}$. Then from (a), and the assumption that $X \times Y$ is a $k$-space, we see that $Y$ does not contain a closed subset homeomorphic to $S_{\omega}$. But $Y$ is the closed image of a metric space, and so by Lemma 2, point-inverses must have compact boundaries. Thus $Y$ is metrizable. If $Y$ were not locally compact, then by Lemmas 3 and $4, X \times Y$ would not be a $k$-space. So $Y$ is locally compact, and (ii) holds. This finishes the proof.

Now we consider a related question. In [ $T_{2}$ ], Tanaka asks the following: Let $Y$ be a closed image, under a map $f$, of a metric space. If $Y^{2}$ is a $k$-space, must each $\partial f^{-1}(y)$ be a Lindelöf space? Tanaka showed in $\left[\mathbf{T}_{3}\right]$ that the answer is yes, assuming $\mathrm{CH}$. By Lemma 2, we see that if some $\partial f^{-1}(y)$ is not Lindelöf, then $Y$ contains a closed copy of $S_{\omega_{1}}$. So we could like to know if $S_{\omega_{1}}^{2}$ is a $k$-space. It turns out that this can be determined in ZFC.

LEMMA 5. $S_{\omega_{1}}^{2}$ is not a $k$-space..

Proof. For each $\alpha \in \omega_{1}$, let $f_{\alpha}: \omega_{1} \rightarrow \omega$ be a function such that $f_{\alpha}$ restricted to $\alpha$ is a one-to-one map onto $\omega$. Define $H_{\alpha}=\left\{\left(m_{\beta}, f_{\alpha}(\beta)_{\alpha}\right) \in S_{\omega_{1}}^{2}: m<f_{\alpha}(\beta)\right\}$, and let $H=\cup_{\alpha<\omega_{1}} H_{\alpha}$.

We claim that $H$ is $k$-closed, but not closed in $S_{\omega_{1}}^{2}$. Suppose $K$ is a compact subset of $S_{\omega_{1}}^{2}$. Then $K$ meets only finitely many sequences in each factor, and thus only finitely many $H_{\alpha}$ 's. Let $\beta(1), \beta(2), \ldots, \beta(n)$ be the sequences in the first factor that $K$ meets. Then $K \cap H_{\alpha}=\left\{\left(m_{\beta(i)}, f_{\alpha}(\beta(i))_{\alpha}\right) \in S_{\omega_{1}}^{2}: m<f_{\alpha}(\beta(i)), i=1\right.$, $2, \ldots, n\}$, which is a finite set. Thus $K \cap H$ is finite and hence closed.

It remains to prove that $H$ is not closed. Let $g: \omega_{1} \rightarrow \omega$, and let $U_{g}$ be the open set in $S_{\omega_{1}}$ containing $(\infty, \infty)$ determined by $g$, as defined in the proof of Lemma 1 . There exists $n_{0} \in \omega$ and an uncountable subset $A$ of $\omega_{1}$, such that $g(\alpha)=n_{0}$ whenever $\alpha \in A$. Let $\gamma$ be an element of $A$ which has infinitely many predecessors in $A$. There exists $\delta \in A$ with $\delta<\gamma$ and $f_{\gamma}(\delta)=m>n_{0}$. Then $\left(m_{\delta}, m_{\gamma}\right) \in H_{\gamma} \cap$ $\left(U_{g} \times U_{g}\right)$. We have shown, then, that $(\infty, \infty)$ must be a limit point of $H$. Therefore, $H$ is not closed and $S_{\omega_{1}}^{2}$ is not a $k$-space.

The following theorem answers the question of Tanaka referred to above.

Theorem 2. Let $Y$ be a closed image, under a map $f$, of a paracompact Fréchet space. If $Y^{2}$ is a $k$-space, then for each $y \in Y, \partial f^{-1}(y)$ is Lindelöf.

Proof. Let $Y$ satisfy the hypotheses. If some $\partial f^{-1}(y)$ is not Lindelö, then by Lemma 2, $Y$ contains a closed copy of $S_{\omega_{1}}$. But $S_{\omega_{1}}^{2}$ is not a $k$-space, so in this case $Y^{2}$ is not a $k$-space.

The next theorem is the same as [ $\mathbf{T}_{2}$, Proposition 2.7], but we do not need the assumption of $\mathrm{CH}$.

THEOREM 3. Let $X$ be a locally compact, paracompact, first countable space, and let $A$ be a closed subset of $X$. Then $(X / \cdot A)^{2}$ is a $k$-space if and only if $\partial A$ is Lindelöf. 
Proof. If $(X / A)^{2}$ is a $k$-space, then $\partial A$ is Lindelöf by Theorem 2. On the other hand, if $\partial A$ is Lindelöf, then Tanaka's proof that $(X / A)^{2}$ is a $k$-space can be used, since this part of his proof does not depend on $\mathrm{CH}$ (or anything else beyond ZFC).

\section{REFERENCES}

[vDW] E. K. van Douwen and M. L. Wage, Small subsets of first countable spaces, Fund. Math. 103 (1979), 103-110.

[T, Y. Tanaka, $A$ characterization for the product of $k$ - and $x_{0}$-spaces and related results, Proc. Amer. Math. Soc. $\$ 9$ (1976), 149-155.

$\left[\mathrm{T}_{2}\right] \ldots$ On the $k$-ness for the products of closed images of metric spaces, General Topology Appl. 9 (1978), 175-183.

$\left[\mathbf{T}_{3}\right] \ldots$, A characterization for the product of closed images of metric spaces to be a $k$-space, Proc. Amer. Math. Soc. 74 (1979), 166-170.

Department of Mathematics, Auburn University, Auburn, Alabama 36830 